\title{
The genus Dentipratulum (Russulales, Auriscalpiaceae): comparative morphology and SEM imaging spore ornamentation split one into three species
}

\author{
Dariusz Karasiński ${ }^{1}$ (1) $\cdot$ Marcin Piątek ${ }^{1}$
}

Received: 27 September 2016 /Revised: 14 December 2016 / Accepted: 16 December 2016 /Published online: 28 December 2016

(C) The Author(s) 2016. This article is published with open access at Springerlink.com

\begin{abstract}
The family Auriscalpiaceae (Russulales) includes species forming pileate, stipitate-pileate, effused-reflexed, and resupinate basidiomata. The resupinate basidiomata are characteristic only for the genus Dentipratulum, described to accommodate Dentipratulum bialoviesense, found in the Białowieża Primeval Forest in Poland and later reported from several locations in Eurasia. The materials assigned to this genus were revised and three distinct morphospecies were detected, including Dentipratulum bialoviesense s. str. and two new species, Dentipratulum khuranae from India and Dentipratulum crystallinum from the Kuril Islands and France. The concept of the genus Dentipratulum is emended to include characters omitted in the original diagnosis (stemlike base of spines, rudimentary subiculum) and found in the novel species (presence of naked or encrusted leptocystidia). All Dentipratulum species are illustrated with pictures of basidiomata, line drawings of micro-morphological characters, and scanning electron microscopy (SEM) micrographs of basidiospores. A key to the genus is provided.
\end{abstract}

Keywords Eurasia $\cdot$ Morphospecies · Taxonomy ·

Wood-inhabiting fungi

Section Editor: Zhu-Liang Yang

Dariusz Karasiński

d.karasinski@botany.pl

1 Department of Mycology, W. Szafer Institute of Botany, Polish Academy of Sciences, Lubicz 46, 31-512 Kraków, Poland

\section{Introduction}

An unusual mucronelloid fungus was found on fallen trunks of Picea abies (L.) H. Karst. in the Białowieża Primeval Forest, in the year 1962, by the Polish mycologist Stanisław Domański. This fungus was similar to the genus Mucronella Fr. but differed in having a gloeopleurous hyphal system with gloeopleurous hyphae and gloeocystidia (lacking in Mucronella), and by having ornamented basidiospores (smooth in Mucronella). Consequently, Domański (1965) described a new genus Dentipratulum Domański and a new species Dentipratulum bialoviesense Domański to accommodate this mucronelloid fungus. Originally, Domański (1965) placed Dentipratulum in the family Hericiaceae, but the presence of sulfocystidia detected by Boidin and Gilles (1990) indicated its affinity to the family Auriscalpiaceae. This family placement was subsequently confirmed in molecular phylogenetic studies (Boidin et al. 1998; Larsson and Larsson 2003; Larsson 2007; Zhou and Dai 2013). The family Auriscalpiaceae (Agaricomycetes, Russulales) includes only four genera: Auriscalpium Gray, Dentipratulum, Gloiodon P. Karst., and Lentinellus P. Karst. (Hibbett et al. 2014), characterized by different morphologies of basidiomata: pileate (Lentinellus), stipitate-pileate (Auriscalpium), effusedreflexed (Gloiodon), and resupinate (Dentipratulum). Except Lentinellus species that have lamellate hymenophores, other members of the family produce hydnoid hymenophores. Macro-morphological similarities of the basidiomata of Dentipratulum and Mucronella are not reflected in their phylogenetic relationships. Both genera are distantly related, and the type species of the genus Mucronella [M. calva (Alb. \& Schwein.) Fr.] belongs to the order Agaricales (Larsson et al. 2004).

After the holotype collection, further specimens of Dentipratulum bialoviesense were only rarely collected. In 
the Białowieża Primeval Forest, it was again found in 1973 by Zdenek Pouzar, labeled as Mucronella sp., and deposited in PRM and KRAM (M. Piatek, unpubl.). The extensive sampling of wood-inhabiting fungi in the Białowieża Primeval Forest in the years 2009-2016, including a large number of mucronelloid specimens, did not yield any additional collection of D. bialoviesense (D. Karasiński, unpubl.). Around the world, Dentipratulum bialoviesense was found only a few times. Corner (1970) reported this fungus from the Kuril Islands (specimen TAAM 12993, collected by Erast Parmasto on Kunashir Island in the year 1960). Later, Boidin and Gilles (1990) and Boidin et al. (1998) added a few specimens of $D$. bialoviesense from France and Switzerland: specimen Gilles 514, collected in 1985 near Pontonx and Buglose (Landes, France); specimen LY 12010, collected by Gérard Gilles in 1986 in Oloron-St Pée (Pyrénées-Atlantiques, France); and specimen LY 15613, collected by M. Meyer in August 1993 in the Canton of Vaud (Switzerland). The ITS sequence of the latter specimen was used in one of the first phylogenetic studies referred to aphyllophoroid fungi (Boidin et al. 1998). One additional specimen was found in 1984 in Landes, Carcen-Ponson, southwestern France (collection Gilles 1645). A duplicate of this material deposited in GB was used as the reference material in molecular phylogenetic studies by Larsson and Larsson (2003) and Larsson (2007).

The herbarium of Stanisław Domański (now preserved in KRAM) contains, except for the holotype material of Dentipratulum bialoviesense, an additional specimen labeled as Dentipratulum sp. originating from India. This specimen was sent to Domański by the Indian mycologist Inder Pal Singh Khurana for his opinion. Judging from the correspondence attached to the specimen, this fungus was considered by Domański as a new species or at least a variety of Dentipratulum bialoviesense. He additionally considered that the Indian specimen may be identical to material reported from the Kuril Islands by Corner (1970). This fungus was never described in the literature. Trying to resolve the enigma of these materials, the revision of all available Dentipratulum specimens appeared to be necessary. This study aims at resolving the taxonomic placement of Dentipratulum specimens by careful morphological analyses using light microscopy (LM) and scanning electron microscopy (SEM), the latter made for the first time for the genus Dentipratulum. The comparative morphology led to the delineation of three morphospecies, Dentipratulum bialoviesense s. str. and two new species. The concept of Dentipratulum is emended.

\section{Materials and methods}

This study is based on specimens deposited in GB, KRAM, LY, and TAAM. Herbarium acronyms are according to Index
Herbariorum (http://sweetgum.nybg.org/ih/). The abbreviation KRAM F-SD refers to the collection of Stanisław Domański preserved in KRAM. All specimens were examined by LM and some specimens also by SEM. For LM, free-hand sections of basidiomata (spines) were made under a Nikon SMZ-2T microscope using a razor blade or single spines were taken and analyzed under a Nikon Eclipse E400 light microscope with magnification up to $1250 \times$. Melzer's reagent, $0.1 \%$ cotton blue in $60 \%$ lactic acid, and $3 \% \mathrm{KOH}$ solution were used as mounting media. Sulfovanillin was used to observe reactions of gloeocystidia and gloeopleurous hyphae. In the species descriptions, spore size range and $Q$ value range are based on 30 measurements per collection, made in Melzer's reagent. The 5\% extreme values of spores are given in parentheses. Twenty measurements were performed for the remaining anatomical elements (basidia, cystidia, and hyphae). Descriptions of macroscopic features of all species were made based on dried specimens and, in the case of Dentipratulum bialoviesense s. str., it was supplemented by characters of fresh basidiomata described in the original diagnosis (Domański 1965). For SEM, single spines from selected specimens (GB-0069354, KRAM F-SD 4001, KRAM F-SD 5881, TAAM 12993) were glued to carbon tabs and fixed to aluminum stubs with double-sided transparent tape. The stubs were sputter-coated with carbon using a Cressington sputter coater and viewed with a Hitachi S-4700 scanning electron microscope, with a working distance of ca. $10 \mathrm{~mm}$. SEM micrographs were taken in the Laboratory of Field Emission Scanning Electron Microscopy and Microanalysis at the Institute of Geological Sciences of the Jagiellonian University, Kraków, Poland. MycoBank (Crous et al. 2004), Index Fungorum (http://www.indexfungorum. org/names/names.asp), and Cortbase (Parmasto et al. 2004) were consulted for nomenclature and abbreviations of authors of fungal names.

\section{Results}

\section{Taxonomy}

Dentipratulum Domański, Acta Mycol. 1: 6, 1965, emend. Karasiński \& Piątek

Basidiomata forming clusters of downwards-growing individual spines, scattered or crowded, pointed and unbranched, often with more or less distinct, short, stem-like base and always with a sterile apex; spines connected with very thin, rudimentary, discontinuous or continuous subiculum. Hyphal system monomitic, generative hyphae with clamp connections, hyaline or rarely brownish in subiculum and at the base of spines, thin to slightly thick-walled. Gloeopleurous hyphae and gloeocystidia present, sulfopositive, usually with strong reaction. Leptocystidia present in some species, fusoid to 
cylindrical, sometimes encrusted. Basidia clavate, constricted to subutriform, sometimes stalked, with four or rarely two sterigmata, with basal clamp. Basidiospores broadly ellipsoidal to globose, 3.5-6 $\mu \mathrm{m}$ in diameter, slightly thick-walled, apiculate, ornamented, strongly amyloid, acyanophilous. Type species: Dentipratulum bialoviesense Domański.

Dentipratulum bialoviesense Domański, Acta Mycol. 1: 7 , 1965, Figs. 1a, 2d, e, 3a, and 4a, b

Type Poland, Podlasie province: Białowieża National Park, on fallen trunk of Picea abies in mixed forest, 6 Aug. 1962, leg. S. Domański (KRAM F-SD 4001, holotype).

Description Basidiomata as individual subtle spines forming on decayed wood linear or irregular aggregations up to 15 long and $5 \mathrm{~cm}$ wide. Spines $0.25-2 \mathrm{~mm}$ long and $75-$ $200 \mu \mathrm{m}$ wide, pointed and unbranched, straight or slightly curved to sinuous, white and soft when fresh, cream ochraceous and brittle when dry, scattered and single or crowded and sometimes with fused bases or occasionally glued laterally, when mature usually with short, stem-like base, 55-90 $\mu \mathrm{m}$ thick and up to $180 \mu \mathrm{m}$ long, sterile at the apex, which consists of projecting hyphal and gloeocystidial ends. Spines connected with very thin, rudimentary and discontinuous subiculum, ca. 10-25 $\mu \mathrm{m}$ thick, relatively distinct around the base of spines, in spaces among spines, and at marginal zones thinning out and disappearing; subiculum is not visible to the naked eye, under a strong lens $(40 \times)$ barely visible as slurred, translucent coat on wood. Hyphal system monomitic, generative hyphae in trama of spines, subhymenium and subiculum $2-4.5(-6) \mu \mathrm{m}$ wide, with clamp connections, hyaline and densely parallelly arranged in trama of spines or slightly brownish and agglutinated in subiculum, thin to slightly thick-walled, inamyloid, acyanophilous. Gloeopleurous hyphae and gloeocystidia present. Gloeopleurous hyphae 2.5-4.5 $\mu \mathrm{m}$, sulfopositive. Gloeocystidia up to $200 \mu \mathrm{m}$ long and 4.5-10 $\mu \mathrm{m}$ wide, usually more or less cylindrical or unevenly swollen in some parts, with obtuse, clavate, narrowly conical, fusiform, or finely papillate apices, sulfopositive, with strong reaction, relatively loosely arranged and scattered in the core of spines, sometimes uncommon, with ends penetrating subhymenium and hymenium, immersed or slightly projecting above basidia, up to $15 \mu \mathrm{m}$. Leptocystidia absent. Basidia 14$20(-25) \times 4-4.8 \mu \mathrm{m}$, variable in shape: clavate and constricted to subutriform, with four or rarely two sterigmata and basal clamp; in hymenium close to the apex of spines basidia with laterally curved, stalked base are often present. Hymenium not thickening. Basidiospores (3.8-)4-5.6(-6) $\times(3.4-) 3.8-$ $5(-5.3) \mu \mathrm{m}(\mathrm{n}=150 / 5), \mathrm{Q}=1.04-1.27$, subglobose to globose, hyaline in $\mathrm{KOH}$, slightly thick-walled, with small but distinct apiculus, verrucose, ornamented with a small,
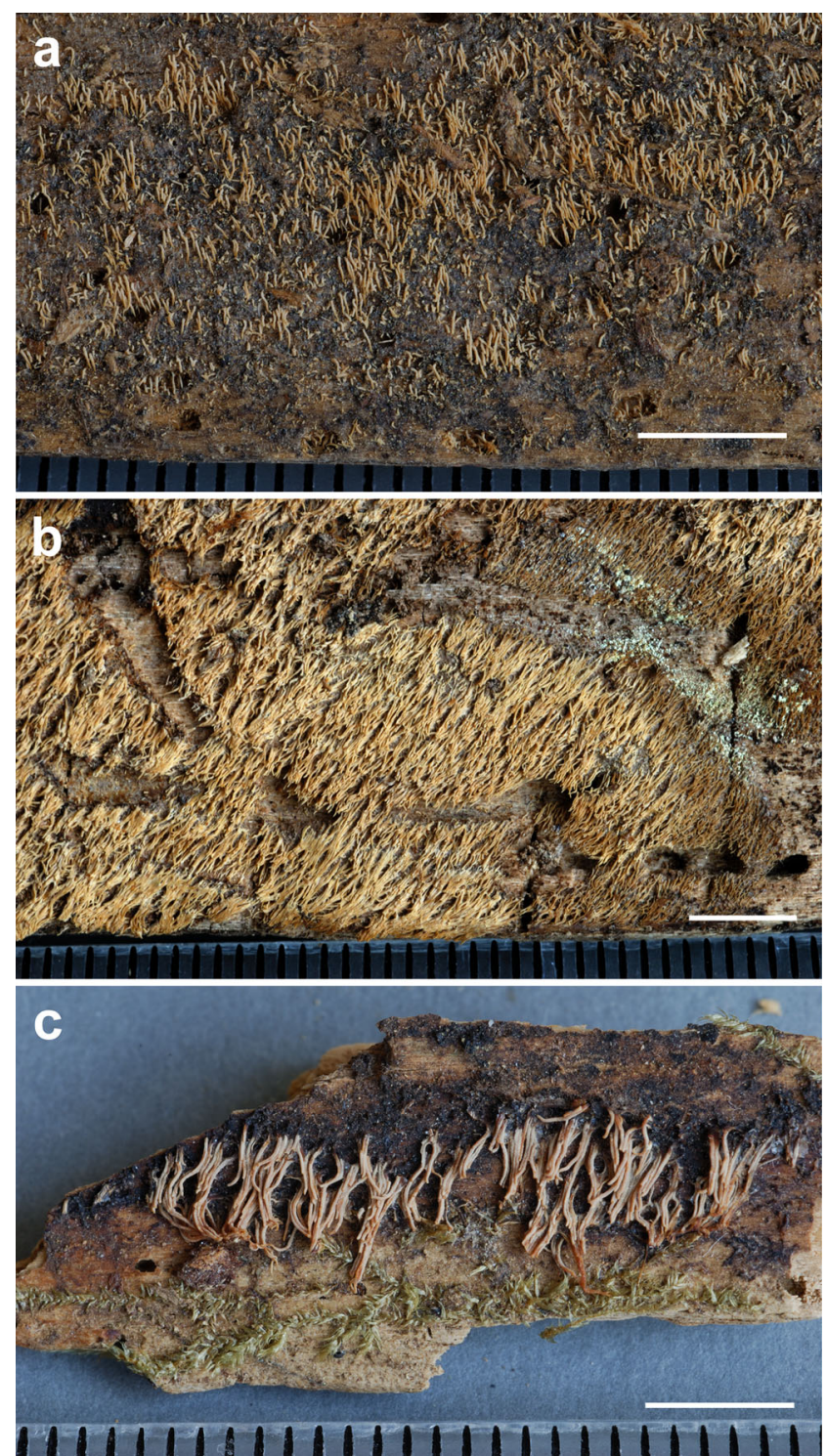

Fig. 1 Dry basidiomata of Dentipratulum species: a Dentipratulum bialoviesense (KRAM F-SD 4001, holotype); b Dentipratulum crystallinum (TAAM 12993, holotype); c Dentipratulum khuranae (KRAM F-SD 5881, holotype). Scale bars $=5 \mathrm{~mm}$

semicircular, or slightly elongated and crowded warts, strongly amyloid, acyanophilous.

Additional specimens examined Poland, Podlasie province: Białowieża National Park, forest section 369, on Picea abies fallen trunk, 27 Aug. 1973, leg. Z. Pouzar (KRAM F-53646, originally labeled as Mucronella sp.). France, PyrénéesAtlantiques: Bonnut, on Quercus robur L., 29 May 1993, leg. G. Gilles 3292 (LY 15557); Pyrénées-Atlantiques: Oloron-St Peé, on unidentified hardwood (Salix?), 17 Aug. 1986, G. Gilles (LY 12010). Switzerland, Bernese Alps, Canton of Vaud: $2 \mathrm{~km}$ of Diablerets, path to Retaud lake, 1300 m a.s.l., on unidentified wood, 26 Sept. 1993, leg. M. Meyer (LY 15613). 

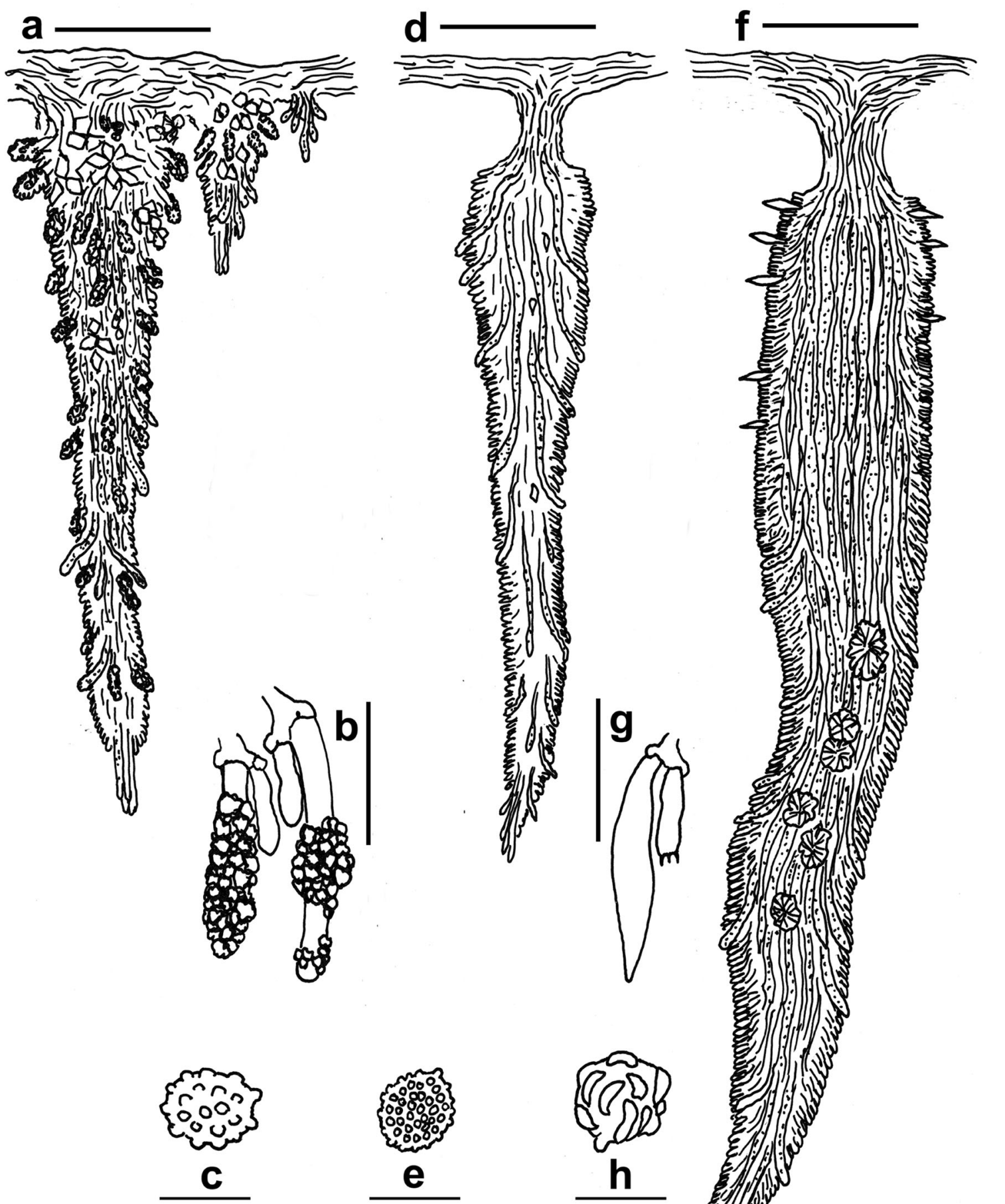

Fig. 2 Schematic line drawings of spines and crucial micromorphological characters of Dentipratulum species: a-c Dentipratulum crystallinum, three spines in various developmental stages, from left to right: mature spine, young spine. and initial spine (a), encrusted

leptocystidia (b), basidiospore (c); $\mathbf{d}$, e Dentipratulum bialoviesense, mature spine (d), basidiospore (e); $\mathbf{f}-\mathbf{h}$ Dentipratulum khuranae, mature spine (f), fusoid leptocystidium (g), basidiospore (h). Scale bars: a, d, $\mathbf{f}=200 \mu \mathrm{m} ; \mathbf{b}, \mathbf{g}=20 \mu \mathrm{m} ; \mathbf{c}, \mathbf{e}, \mathbf{h}=5 \mu \mathrm{m}$

Distribution and ecology This is a very rare European species, and according to revised material, it is known only from four localities in Poland, France, and Switzerland. It is

associated with decayed wood of conifers and hardwoods in medium or late stages of decomposition. Both specimens known from Białowieża Primeval Forest were collected from 


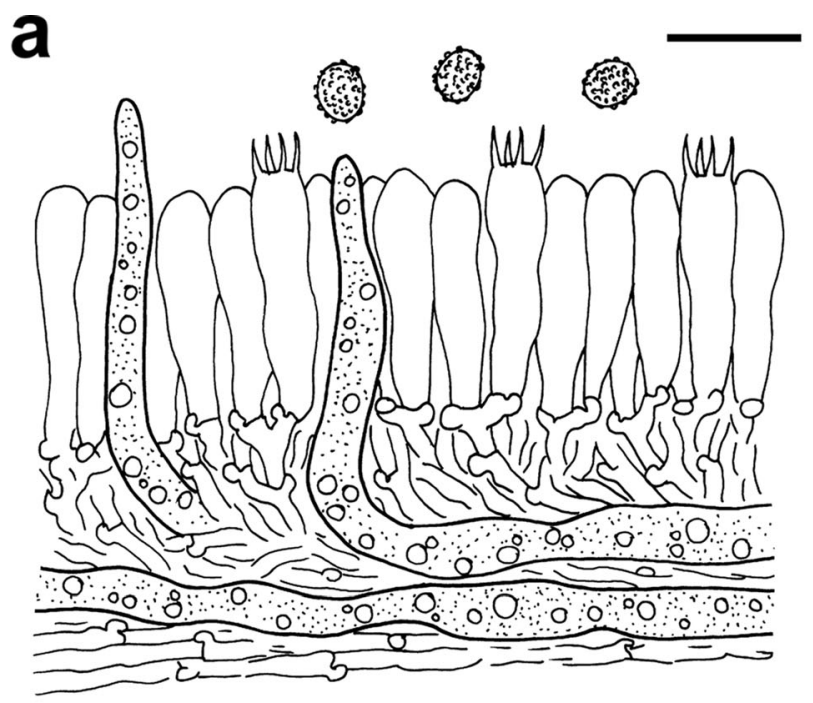

Fig. 3 Hymenia and spine tramas of Dentipratulum species in vertical section showing arrangements of microscopical structures: a Dentipratulum bialoviesense (KRAM F-SD 4001, holotype); b Dentipratulum crystallinum (TAAM 12993, holotype); c Dentipratulum khuranae (KRAM F-SD 5881, holotype). Scale bars $=10 \mu \mathrm{m}$

fallen trunks of Picea abies lying on the ground in mixed lowland forest of primeval origin. Outside Poland, Dentipratulum bialoviesense is known from mountain locations in southwestern France and Switzerland on Quercus robur and unidentified wood, respectively.

Dentipratulum crystallinum Karasiński, sp. nov., Figs. 1b, 2a, b, c, 3b, and 4c, d

MycoBank MB 819474

Diagnosis Differs from Dentipratulum bialoviesense by having slightly narrower basidiospores ornamented with larger and more scattered warts visible in SEM, and presence of cylindrical, hyphoid, often projecting leptocystidia, obtuse at the apex, basally clamped, more or less covered with crystalline matter. In old basidiomata, this species forms also crystalline matter on some gloeocystidial apices.

Etymology The specific epithet refers to encrusted leptocystidia and gloeocystidia.

Type Russia. Kuril Islands: Kunashir Island, Alyokhino, on a fallen trunk of Betula ermanii Cham. in a nemoral forest, 5 Oct. 1960, leg. E. Parmasto (TAAM 12993, holotype).

Description Basidiomata forming irregular patches up to $4 \mathrm{~cm}$ long and $2.5 \mathrm{~cm}$ wide, consisting of pointed downwards spines, initially scattered, finally crowded, connected with a thin subiculum, 20-80 $\mu \mathrm{m}$ thick. Single spines $0.15-2 \mathrm{~mm}$ long and up to $200 \mu \mathrm{m}$ thick, starting as conical pegs of gloeocystidia, when mature sessile, without stem-like base, conical to subcylindrical, with sterile apex consisting of single gloeocystidial ends and projecting hyphal ends sometimes encrusted with small subhyaline crystals; spines whitish to cream ochraceous when dry, under lens pruinose due to projecting, encrusted leptocystidia, which are present especially at the base of young spines. Hyphal system monomitic, generative hyphae in trama of spines, subhymenium and subiculum 1.5-4.5(-6.5) $\mu \mathrm{m}$ wide, with clamp connections, thin-walled in trama and subhymenium, slightly thick-walled in a basal part of spines, densely arranged; subicular hyphae 1.5-2.5 $\mu \mathrm{m}$ wide, thin-walled, agglutinated, hyaline to yellow brown due to resinous content; single, rhomboid crystals or their agglomerations often present amongst hyphae and on the surface of spines. Gloeopleurous hyphae 2.5-4 $\mu \mathrm{m}$, sulfopositive, hardly differentiated from gloeocystidia. Gloeocystidia numerous, sulfopositive with strong reaction, up to $220 \mu \mathrm{m}$ long and 4-10 $\mu \mathrm{m}$ wide, cylindrical or with a 

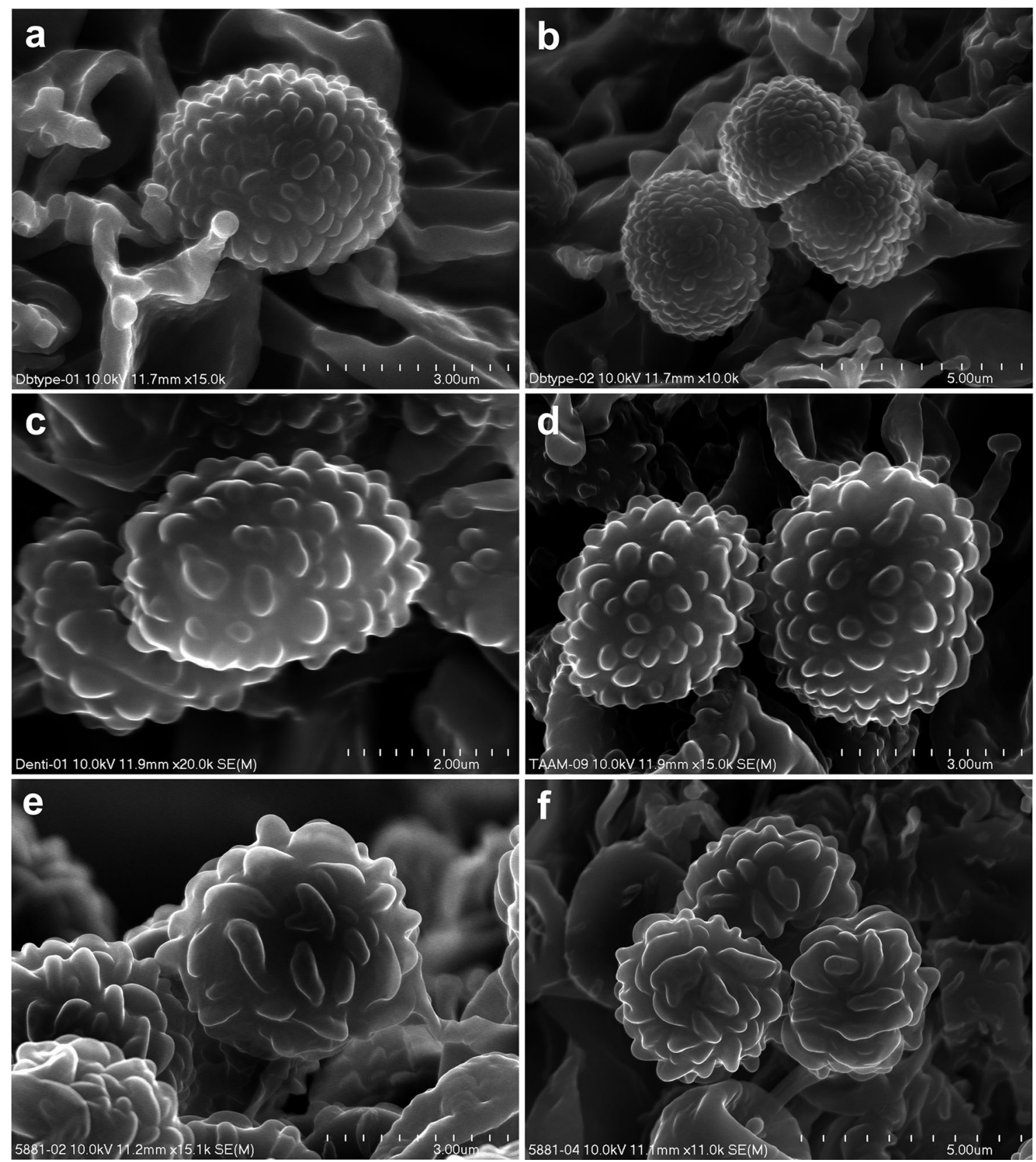

Fig. 4 Basidiospores of Dentipratulum species as seen by scanning electron microscopy (SEM): a, b Dentipratulum bialoviesense (KRAM F-SD 4001, holotype); c Dentipratulum crystallinum (GB-006 9354); d

Dentipratulum crystallinum (TAAM 12993, holotype); e, f Dentipratulum khuranae (KRAM F-SD 5881, holotype). Scale bars: a, $\mathbf{d}, \mathbf{e}=3 \mu \mathrm{m} ; \mathbf{b}, \mathbf{f}=5 \mu \mathrm{m} ; \mathbf{c}=2 \mu \mathrm{m}$

few inflations in the middle part, occasionally with lateral outgrowth close to the base, protruding into hymenium, with sinuous-cylindrical, fusiform, or rarely papillate ends, immersed or projecting above hymenial layer up to $10 \mu \mathrm{m}$, with yellowish or hyaline granular contents; gloeocystidia together with gloeopleurous hyphae form distinct core of spines. Leptocystidia $20-40 \times 3-4.5 \mu \mathrm{m}$, cylindrical, obtuse at the apex, thin-walled, with basal clamps, more or less encrusted with subhyaline matter; abundant especially at the base of spines in young basidiomata, and then distinctly projecting, but numerous also in mature basidiomata, and then immersed or only slightly projecting above basidia. Basidia 13-25 × $5 \mu \mathrm{m}$, subclavate, and constricted to suburniform, in hymenium close to top of spines often with curved and slightly stalked base, basally clamped, with four sterigmata up to $5 \mu \mathrm{m}$ long. Basidiospores (4-)4.2-5.4(-5.6) $\times(3.6-) 3.8-$ 4.5(-4.8) $\mu \mathrm{m}(\mathrm{n}=60 / 2), \mathrm{Q}=1.1-1.31$, broadly ellipsoidal to subglobose, with distinct apiculus, hyaline in $\mathrm{KOH}$, slightly thick-walled, verrucose, ornamented with relatively large and distant semicircular warts, strongly amyloid, acyanophilous. 
Additional specimen examined France, Aquitaine: Landes, Carcen-Ponson, on wood of Salix atrocinerea Brot., 21 Sept. 1984, leg. G. Gilles 1645 (GB-006 9354).

Distribution and ecology This is a Eurasian species, currently known from the Kuril Islands and southwestern France. It is an inhabitant of decayed wood of deciduous trees, namely Betula ermanii and Salix atrocinerea.

Comments The type specimen from the Kuril Islands represents a mature basidiome with crowded, up to $2 \mathrm{~mm}$ long spines, encrusted leptocystidia, and abundant apically encrusted gloeocystidia. The specimen GB-006 9354 from France differs from the type in that: the spines are scattered and short, up to $0.7 \mathrm{~mm}$ long, and crystalline matter on the gloeocystidial apices is absent. However, the basidiospore ornamentation pattern is identical as in the type, and encrusted leptocystidia are abundantly present. Therefore, it is likely that this specimen is not fully mature and represents an initial stage of development of Dentipratulum crystallinum. The 5.8SITS2-28S nuclear rDNA sequence deposited in GenBank as Dentipratulum bialoviesense under accession number AF506389 was obtained from this specimen and used in some molecular phylogenetic studies (Larsson and Larsson 2003; Larsson 2007; Zhou and Dai 2013).

Dentipratulum khuranae Karasiński \& Piątek, sp. nov., Figs. 1c, 2f, g, h, 3c, and 4e, f MycoBank MB 819475

Diagnosis Differs from Dentipratulum bialoviesense and D. crystallinum by having larger spines up to $3.8 \mathrm{~mm}$ long and up to $300 \mu \mathrm{m}$ wide, presence of fusoid leptocystidia, and globose basidiospores ornamented with relatively large, short ridges.

Etymology This species is named in honor of the Indian mycologist Inder Pal Singh Khurana, who collected the new species.

Type India, Himachal Pradesh: Shimla District, in the vicinity of Baghi village, on wood of a fallen log in a mixed forest (Picea A. Dietr., Abies Mill., and scattered Juglans L.), 1976, leg. I.P.S. Khurana (KRAM F-SD 5881, holotype).

Description Basidiomata forming small, linear patches $2 \mathrm{~cm}$ long and $0.5 \mathrm{~cm}$ wide, consisting of single or fasciculate spines arising from very thin subiculum, almost invisible to the naked eye. Single spines up to $3.8 \mathrm{~mm}$ long and up to $300 \mu \mathrm{m}$ wide, whitish to cream ochraceous when dry, with stem-like base, $80-140 \mu \mathrm{m}$ thick and up to $200 \mu \mathrm{m}$ long, subulate or obtuse at the sterile apex, which consists of projecting hyphal and gloeocystidial ends. Hyphal system monomitic, generative hyphae in trama of spines, subhymenium and subiculum 2-5 $\mu \mathrm{m}$ wide, with clamp connections, not inflated, thin to slightly thick-walled close to the base and in stem-like part; gloeopleurous hyphae, 2.5 $5.5 \mu \mathrm{m}$ wide, present abundantly in trama, extending in the hymenium as gloeocystidia, often irregularly swollen up to $10 \mu \mathrm{m}$, sulfopositive with strong reaction, turning black in sulfobenzaldehyde, acyanophilous. Trama consisting of generative hyphae, gloeopleurous hyphae, elongate gloeocystidia and sometimes large crystals, circular or ellipsoidal in outline, ca. $40 \mu \mathrm{m}$ in diam. Hymenium thickening, up to $75 \mu \mathrm{m}$, comprising gloeocystidia, basidia, and rare leptocystidia. Leptocystidia $19-45 \times 3.5-5 \mu \mathrm{m}$, fusoid, subulate, thinwalled, projecting up to $15 \mu \mathrm{m}$ above basidia; usually uncommon and occur especially at the base of spines. Gloeocystidia often longer than $250 \mu \mathrm{m}, 5-10(-12) \mu \mathrm{m}$ wide, projecting up to $20 \mu \mathrm{m}$ above the hymenium. The distribution of gloeocystidia and leptocystidia uneven, leptocystidia sometimes abundant toward the base but usually uncommon, while gloeocystidia abundant toward the spine apices. Basidia 18-27 × 4-5 $\mu \mathrm{m}$, variable in shape, clavate-cylindric, constricted, often subutriform, clamped at the base, with four sterigmata. Basidiospores $(3.8-) 4.3-5.4(-5.8) \times(4-) 4.2-5.2(-5.4) \mu \mathrm{m}(\mathrm{n}=30 / 1)$, $\mathrm{Q}=1.02-1.12$, globose to subglobose, slightly thick-walled, ornamented with irregular, short, and distant ridges, with small apiculus, hyaline in $\mathrm{KOH}$, strongly amyloid, acyanophilous.

Distribution and ecology This species is known only from the type locality in northwestern India in Asia. The specimen was collected from decayed wood of an unidentified tree in mixed forest with Picea, Abies, and Juglans.

\section{Discussion}

The re-examination of the holotype material of Dentipratulum bialoviesense and remaining materials assigned here to the genus Dentipratulum resulted in the detection of some features, such as subiculum, stem-like base of spines, and leptocystidia, which were not included in the original diagnosis of the genus (Domański 1965). Therefore, the concept of the genus is emended to include these characters. A thin subiculum is constantly present in all accepted species of Dentipratulum. This character was already observed by Corner (1970) in specimen TAAM 12993 originally referred to $D$. bialoviesense but representing $D$. crystallinum. The subiculum is rudimentary and discontinuous in D. bialoviesense, slightly thicker, and more distinct in $D$. khuranae and the thickest in D. crystallinum. The spines with stem-like bases are present in D. bialoviesense and $D$. khuranae but absent in D. crystallinum. The spines of D. bialoviesense and D. crystallinum are similar in size, up to $2 \mathrm{~mm}$ long and $200 \mu \mathrm{m}$ wide, while $D$. khuranae produces spines significantly longer and wider, up to $3.8 \mathrm{~mm}$ long and $300 \mu \mathrm{m}$ wide. The leptocystidia are present in D. crystallinum 
and $D$. khuranae, though they are lacking in the generic type. The leptocystidia in D. crystallinum and D. khuranae differ from each other by shape and occurrence of encrustation. The distinction between leptocystidia and gloeocystidia is not always clear, but leptocystidia lack the sulfopositive reaction and have hymenial origin, in contrary to the tramal origin of gloeocystidia.

The basidiospores observed in LM are very similar in shape and size in all Dentipratulum species, although slight differences could be detected in the $\mathrm{Q}$ value ranges. These values are almost the same for D. bialoviesense and D. crystallinum (1.04-1.27 vs. 1.1-1.31, respectively), but different in D. khuranae $(\mathrm{Q}=1.02-1.12)$. Moreover, the basidiospores of $D$. khuranae have a more globose appearance than in remaining species, and $D$. crystallinum has more ellipsoidal (narrower) basidiospores than D. bialoviesense. The ornamentation pattern of basidiospores observed in LM is roughly similar in all species but clearly different, as observed by SEM. The ornamentation pattern is more similar between $D$. bialoviesense and $D$. crystallinum than in D. khuranae, but is specific to each of them. The basidiospores of D. bialoviesense have low, small, and crowded warts, while the basidiospores of $D$. crystallinum have distinctly larger warts that are more distantly spaced from each other. The basidiospores of D. khuranae have ornamentation in the form of irregular, short, and distant ridges.

Except for these morphological characters, the minor differences could be observed in the gloeopleurous hyphal system. In Dentipratulum crystallinum and D. khuranae, gloeocystidia and gloeopleurous hyphae are densely arranged and form a distinct core of spines. In $D$. bialoviesense, gloeocystidia are usually somewhat loosely arranged and more scattered in trama of spines; however, dense configuration is occasionally observed also in this species. Additionally, in D. bialoviesense and $D$. khuranae, gloeocystidia are always naked, in contrast to gloeocystidia of $D$. crystallinum, which are often apically covered by fine crystals in mature specimens.

In summary, the main differentiating characters in the genus Dentipratulum are: (1) the type of basidiospore ornamentation observed in SEM; (2) the occurrence, shape, and encrustation of hymenial leptocystidia; (3) the configuration of gloeopleurous hyphae and gloeocystidia; (4) the morphology and size of spines.

\section{A key to the Dentipratulum species}

1. Leptocystidia absent. D. bialoviesense $1 *$ Leptocystidia present 2

2. Leptocystidia cylindrical and obtuse at apex, encrusted; spines up to $2 \mathrm{~mm}$ long, without stem-like base; basidiospore walls covered with semicircular warts D. crystallinum

2*. Leptocystidia fusoid and naked; spines up to $3.8 \mathrm{~mm}$ long, with stem-like base; basidiospore walls covered with irregular, short, and distant ridges.

D. khuranae

Acknowledgments We thank the curators of GB, LY, and TAAM for the loan of specimens, and Anna Łatkiewicz (Kraków) for her help with the SEM micrographs. This work was supported by the statutory funds of the W. Szafer Institute of Botany, Polish Academy of Sciences, Kraków, Poland.

Open Access This article is distributed under the terms of the Creative Commons Attribution 4.0 International License (http:// creativecommons.org/licenses/by/4.0/), which permits unrestricted use, distribution, and reproduction in any medium, provided you give appropriate credit to the original author(s) and the source, provide a link to the Creative Commons license, and indicate if changes were made.

\section{References}

Boidin J, Gilles G (1990) Corticiés s.l. intéressants ou nouveaux pour la France (Basidiomycotina). Bull Soc Mycol Fr 106:135-167

Boidin J, Mugnier J, Canales R (1998) Taxonomie moleculaire des Aphyllophorales. Mycotaxon 66:445-491

Corner EJH (1970) Supplement to "A Monograph of Clavaria and allied genera". Beih Nova Hedwigia 33:1-299

Crous PW, Gams W, Stalpers JA, Robert V, Stegehuis G (2004) MycoBank: an online initiative to launch mycology into the 21st century. Stud Mycol 50:19-22

Domański S (1965) Wood-inhabiting fungi in Białowieża virgin forest in Poland. II. The mucronelloid fungus of the Hericiumgroup: Dentipratulum bialoviesense, gen. et sp. nov. Acta Mycol 1:5-11

Hibbett DS, Bauer R, Binder M, Giachini AJ, Hosaka K, Justo A, Larsson E, Larsson KH, Lawrey JD, Miettinen O, Nagy LG, Nilsson RH, Weiss M, Thorn RG (2014) Agaricomycetes. In: McLaughlin DJ, Spatafora JW (eds) The Mycota. VII Part A. Systematics and evolution, 2nd edn. Springer-Verlag, Berlin, pp 373-429

Larsson KH (2007) Re-thinking the classification of corticioid fungi. Mycol Res 111:1040-1063

Larsson E, Larsson KH (2003) Phylogenetic relationships of russuloid basidiomycetes with emphasis on aphyllophoralean taxa. Mycologia 95:1037-1065

Larsson KH, Larsson E, Kõljalg U (2004) High phylogenetic diversity among corticioid homobasidiomycetes. Mycol Res 108:983-1002

Parmasto E, Nilsson RH, Larsson KH (2004) Cortbase version 2 - extensive updates of a nomenclatural database for corticioid fungi (Hymenomycetes). Phyloinformatics 5:1-7

Zhou LW, Dai YC (2013) Taxonomy and phylogeny of wood-inhabiting hydnoid species in Russulales: two new genera, three new species and two new combinations. Mycologia 105:636-649 